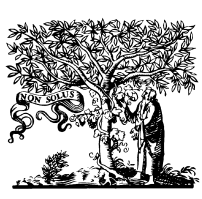

ELSEVIER

\title{
Probabilistic seismic hazard maps for the Japanese islands
}

\author{
A. Kijko ${ }^{\mathrm{a}}$, A.O. Öncel ${ }^{\mathrm{b}, *}$ \\ ${ }^{a}$ Council for Geoscience, Geological Survey of South Africa, Private Bag X112, Pretoria, 0001 South Africa \\ ${ }^{\mathrm{b}}$ Department of Geophysical Engineering, Division of Seismology, Istanbul University, 34850 Istanbul, Turkey
}

\begin{abstract}
Probabilistic seismic hazard maps are constructed by utilising a new methodology called the parametric-historic method, since it combines the best features of the 'deductive' (Cornell CA. Engineering seismic risk analysis. Bull Seism Soc Am 1968;58;1583-606) and 'historic' (Veneziano D, Cornell CA, O’Hara T. Historic method for seismic hazard analysis. Elect Power Res Inst, Report NP-3438, Palo Alto, 1984) procedures.

The technique has been developed specifically for the estimation of seismic hazard at individual sites without the subjective judgment involved in the definition of seismic source zones, when specific active faults have not been mapped or identified, and when the causes of seismicity are not well understood. The combination of historical and instrumental data is permitted. The historical part of the catalogue contains only the strongest events, whereas the complete part can be divided into several subcatalogues, each assumed complete above a specified threshold of magnitude. Uncertainty in the determination of magnitude has also been taken into account. The maximum credible magnitude, $m_{\max }$, is of paramount importance in this approach.

The seismic hazard maps are based on a long-term earthquake history (599-1997) compiled of the catalogues of Utsu (Utsu T. Catalog of large earthquakes in the region of Japan from 1885 through 1980. Bull Earthq Res Inst, Univ Tokyo, 1982;57:401-63), Usami (Usami T. Materials for comprehensive list of destructive earthquakes in Japan. Tokyo: Tokyo Press, 1996) and JMA for the Japanese islands. The analysis is based on subregions at a grid size of $0.05^{\circ}$ along the Japanese islands, for each of which peak ground accelerations and spectral accelerations for natural frequencies of $1,3,5$ and $10 \mathrm{~Hz}$, are predicted and mapped to occur at a $10 \%$ probability in 50 years. (C) 2001 Published by Elsevier Science Ltd.
\end{abstract}

\section{Introduction}

Following McGuire [1], the existing procedures of probabilistic seismic hazard analysis (PSHA) fall into two main categories: deductive and historic.

The theoretical basis for the deductive method was formulated by Cornell [2]. Application of the procedure includes several steps. The initial step requires the definition of potentially active seismic regions (zones). In the next step, seismicity parameters (usually mean seismic activity rate $\lambda$, the level of completeness of the earthquake catalogue $m_{\min }$, the maximum earthquake magnitude $m_{\max }$, and the Gutenberg-Richter parameter $b$ ) are determined for each seismogenic zone. Assessment of these parameters requires a declustered seismic event catalogue containing origin times, size of seismic events and spatial locations [3]. The following step involves the selection of a ground-motion relation giving the cumulative distribution function $(\mathrm{CDF})$

\footnotetext{
* Corresponding author. Tel.: +90-212-5938237/262; fax: +90-2125911997.

E-mail address: aoncel@zdnetonebox.com (A.O. Öncel).
}

for a required ground motion parameter. The final step requires the integration of individual contributions from each seismogenic zone into a site-specific distribution.

The major disadvantage of any deductive-type procedure of PSHA stems from the requirement of the specification of the seismogenic source zones. Often tectonic provinces or specific active faults have not been identified and mapped, and causes of seismicity are not well understood.

The second category of PSHA, the so-called historic methods [4], requires input data about past seismicity only, and does not require specification of seismogenic zones. For each historic earthquake, the empirical distribution of the required seismic hazard parameter is estimated by using its value of magnitude, epicentral distance and assumed ground motion attenuation relation. By normalizing this distribution for the duration of the seismic event catalogue, one obtains an annual rate of exceedance of a predetermined value of the hazard parameter. The major disadvantage of the procedure is its poor reliability in estimating small probabilities for areas of low seismicity. The procedure is not recommended for an area where the seismic event catalogues are highly incomplete. 
Bearing in mind both the weak and strong points of the above two approaches, an alternative procedure [5-7] was proposed, which could be classified as a parametric-historic approach. The new approach combines the best of the deductive and historic procedures and, in many cases, is free from the basic disadvantages characteristic of each of the procedures. Since in the proposed approach the maximum regional magnitude, $m_{\max }$, is of paramount importance, the authors present a statistical technique that can be used for evaluation of this important parameter.

This paper examines spatial variations in the ground motion characteristics of seismicity and hazard acceleration response spectra for different natural frequencies. Events in the Japanese islands over a period from 599 to 1997 are considered in the analysis.

\section{Probabilistic procedure for estimation of maximum regional magnitude $m_{\max }$}

Suppose that in the area of concern, within a specified time interval $T$, there are $n$ main seismic events with magnitudes $M_{1}, M_{2}, \ldots, \mathrm{Mn} . \quad$ Each magnitude $M_{i} \geq m_{\min }$ $(i=1, \ldots, n)$, where $m_{\min }$ is a known threshold of completeness (i.e. all events having magnitude greater than or equal to $m_{\min }$ are recorded). It is further assumed that the seismic event magnitudes are independent, identically distributed, random values with probability density function, PDF, equal to $f_{\mathrm{M}}\left(m \mid m_{\max }\right)$ and cumulative distribution function, $\mathrm{CDF}$, equal to $F_{\mathrm{M}}\left(m \mid m_{\max }\right)$. Parameter $m_{\max }$ is the upper limit of the range of magnitudes and thus denotes the unknown maximum regional magnitude, which is to be estimated. For the Bayesian version of the Gutenberg-Richter frequency-magnitude relation, where the uncertainty of its parameter $\beta$ is taken into account, the respective CDF, $F_{M}\left(m \mid m_{\max }\right)$, of earthquake magnitude is 0 for $m \leq m_{\min }, 1$ for $m \geq m_{\max }$, and

$F_{M}\left(m \mid m_{\max }\right)=C_{\beta}\left\{1-\left[p /\left(q+m-m_{\min }\right)\right]^{q}\right\}$

for $m_{\min }<m<m_{\max } . C_{\beta}$ is a normalising coefficient equal to $1 /\left\{1-\left[p /\left(p+m_{\max }-m_{\min }\right)\right]^{q}\right\}$, where parameters $p$ and $q$ can be expressed through the expected value and variance of the $\beta$-value, $p=\bar{\beta} /\left(\sigma_{\beta}\right)^{2}, q=\left(\bar{\beta} / \sigma_{\beta}\right)^{2}, \beta=b \ln (10)$, and $b$ is the $b$-parameter of the Gutenberg-Richter relation. Eq. (1) is known also as the Bayesian Exponential-Gamma $\mathrm{CDF}$ of earthquake magnitude.

From the condition that compares the largest observed magnitude $m_{\max }^{o b s}$ and the maximum expected magnitude during a specified time interval $T$, the maximum regional magnitude $m_{\max }$ is [4]

$\hat{m}_{\max }=m_{\max }^{\mathrm{obs}}+\int_{\mathrm{m}_{\min }}^{m_{\max }^{\mathrm{obs}}}\left[F_{M}\left(m \mid m_{\max }^{\mathrm{obs}}\right)\right]^{n} \mathrm{~d} m$.

It is not difficult to show that after application of Cramer's approximation, the estimator of the maximum regional magnitude $m_{\max }$ and its approximate variance becomes

$$
\begin{aligned}
& \hat{m}_{\max }= \\
& \quad m_{\max }^{\text {obs }}+\frac{\delta^{-1 / q+2} \exp \left[n r^{q} /\left(1-r^{q}\right)\right]}{\beta}\left[\Gamma\left(-1 / q, \delta r^{q}\right)-\Gamma(-1 / q, \delta)\right],
\end{aligned}
$$

$\operatorname{Var}\left(\hat{m}_{\max }\right)=$

$$
\sigma_{M}^{2}+\left\{\frac{\delta^{-1 / q+2} \exp \left[n r^{q} /\left(1-r^{q}\right)\right]}{\beta}\left[\Gamma\left(-1 / q, \delta r^{q}\right)-\Gamma(-1 / q, \delta)\right]\right\}^{2},
$$

where $\delta=n C_{\beta}$ and $\Gamma(\cdot, \cdot)$ is the incomplete gamma function. The maximum likelihood procedure for the assessment of the area-specific mean seismic activity rate, $\lambda_{A}$, and the Gutenberg-Richter parameter, $\beta$, is described in [7]. Extensive analysis of performance of the above estimators is given in [4].

\section{Assessment of seismic hazard for a given site}

To express seismic hazard in terms of peak ground acceleration (PGA), the aim would be to calculate the conditional probability that a single earthquake of random magnitude, $M$, at a random distance, $R$, will cause a PGA equal to, or greater than, an acceleration of engineering interest $a_{\min }$. For this purpose the assumption is made that in the range of interest the attenuation curve of the PGA, $a$, has the following form:

$\ln (a)=c_{1}+c_{2} M+\phi(R)+\epsilon$,

where $c_{1}$ and $c_{2}$ are empirical constants, $M$ is the earthquake magnitude, and $\phi(R)$ is a function of earthquake distance $R$. The term $\epsilon$ is a random error, which has been observed to have a normal (Gaussian) distribution. Usually $\phi(R)$ is of the form $c_{3} R+\mathrm{c}_{4} \ln (R)$, where $c_{3}$ and $c_{4}$ are empirical constants. An application of a straightforward approach, like Cornell's [2], implies that after normalization, the $\mathrm{CDF}$ of the logarithm of $\mathrm{PGA}$ is:

$F_{X}\left(x \mid x_{\max }\right)=\frac{\exp \left(-\gamma x_{\min }\right)-\exp (-\gamma x)}{\exp \left(-\gamma x_{\min }\right)-\exp \left(-\gamma x_{\max }\right)}$,

where $x=\ln (a), \gamma=\beta / c_{2}, x_{\min }=\ln \left(a_{\min }\right), x_{\max }=\ln \left(a_{\max }\right)$, and $a_{\max }$ is the maximum possible PGA at the site. From Eq. (6), it follows that at any given site the logarithm of the PGA is distributed according to the same type of distribution as earthquake magnitude, i.e. negative exponentialthe form of the familiar Gutenberg-Richter distribution. The CDF of the logarithm of the largest PGA observed at the site is doubly truncated. The first truncation from the bottom, $x_{\min }=\ln \left(a_{\min }\right)$, represents the chosen threshold of acceleration of engineering interest. The second truncation, $x_{\max }=\ln \left(a_{\max }\right)$, is an unknown parameter representing the logarithm of the maximum possible PGA at the site. 
Therefore, for a given $a_{\min }$, the seismic hazard at the site is determined by three parameters: $\lambda, \gamma$ and $a_{\max }$. In order to estimate these parameters, the largest PGAs, $a_{1}, \ldots, a_{m}$, felt at the site of interest are selected from $m$ consecutive time intervals, $t_{1}, \ldots, t_{m}$, and the maximum likelihood procedure is used.

\section{Assessment of the response spectra of acceleration at the site}

Let us assume that the regression coefficients $c_{1}, \ldots, c_{4}$ for the attenuation Eq. (5) are available for different ground motion frequencies of acceleration response spectra. Therefore, for a given frequency $f_{i}$ of ground motion, the amplitude of spectral acceleration (SA) $a$ $\left(f_{i}\right)$ is described by

$\ln \left[a\left(f_{i}\right)\right]=c_{1 i}+c_{2 i} M+c_{3 i} R+c_{4 i} \ln (R)+\epsilon$,

where $c_{j i}$ are known regression coefficients, $i=1, \ldots, n_{\mathrm{f}}$, $j=1, \ldots, 4$, and $n_{\mathrm{f}}$ is the number of selected frequencies in the SA. In addition it is assumed that the spectral amplitude $a\left(f_{\mathrm{i}}\right) \geq a_{\min }$ for all $i$, where $a_{\min }$ denotes the minimum value of the SA of engineering interest. Let $\lambda_{i} \equiv \lambda\left(x_{i}=x_{\min }\right)$ denotes the unknown mean activity rate of occurrence of events giving rise to a SA at frequency $f_{i}, x_{i}$, with $\mathrm{xi} \geq x_{\min }=$ $\ln \left(a_{\mathrm{min}}\right)$, i.e. when the SA amplitude of the ground motion at the site $a\left(f_{\mathrm{i}}\right) \geq a_{\min }$. Following the same approach as applied for the PSHA, expressed in terms of PGA, the mean activity rate of events with $x_{i} \geq x_{\min }$, is equal to $\lambda\left(x_{\mathrm{i}}\right)=\lambda_{\mathrm{i}}\left[1-F_{X_{i}}\left(x_{\mathrm{i}} \mid x_{\max (i)}\right)\right]$, where $x_{\max (i)}=\operatorname{Len}\left(a_{\max (i)}\right)$ and $a_{\max (i)}$ is the maximum possible amplitude of SA at frequency $f_{\mathrm{i}}$. The function $F_{X_{i}}\left(x_{i} \mid x_{\max (i)}\right)$ denotes the CDF

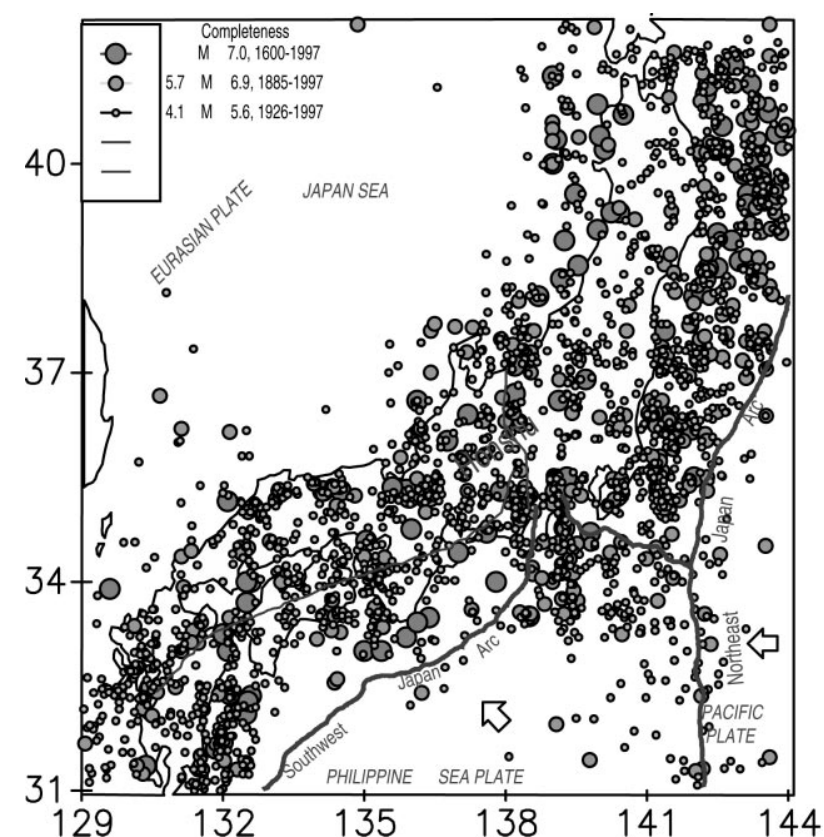

Fig. 1. Seismicity map for the Japanese islands and surrounding region. FTJ, Fuji Triple Junction; BTJ, Boso triple Junction.

of the logarithm of SA $a\left(f_{i}\right)$ [Eq. (7)], where parameter $\gamma$ is replaced by $\gamma_{i}=\beta / c_{2 i}$ and $c_{2 i}$ is the corresponding regression coefficient in the attenuation Eq. (5). From the same procedure as applied for PGA, all spectral characteristics of seismic hazard can be obtained from the $n_{\mathrm{f}}$ values of mean activity rates $\lambda_{i}\left(i=1, \ldots, n_{\mathrm{f}}\right)$ and the parameter $\beta$ of the Gutenberg-Richter relation. The maximum likelihood estimates of parameters $\lambda_{i}$ and $\beta$ are the values of $\hat{\lambda}_{i}$ and $\hat{\beta}$ that

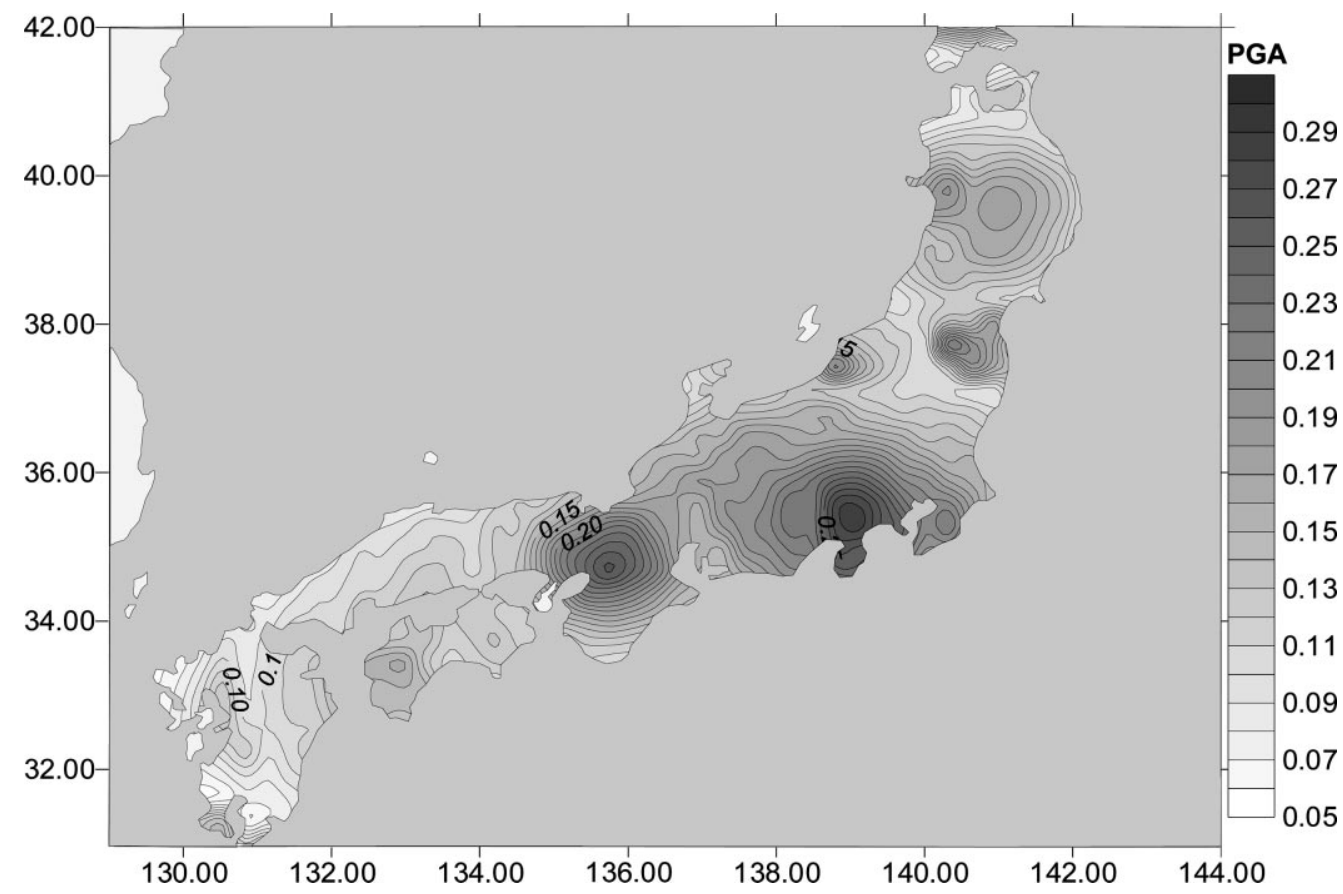

Fig. 2. Peak ground acceleration (g) contour lines for the Japanese islands at a $10 \%$ probability to occur in 50 years. 

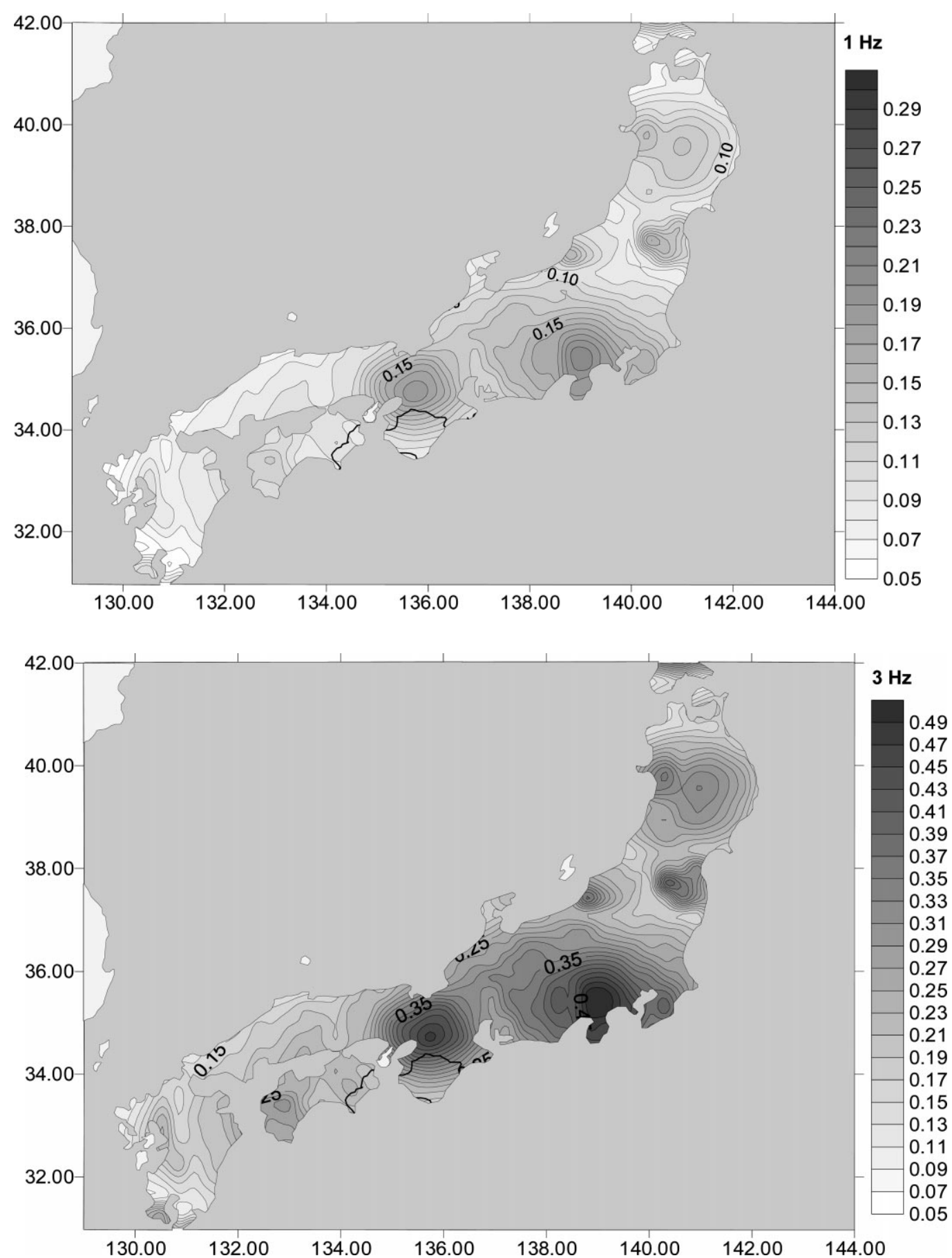

Fig. 3. The maps of the horizontal acceleration response spectra (5\% damping, for sites of hard rock) for natural frequencies: (a) 1 ; (b) 3 ; (c) 5 ; and (d) $10 \mathrm{~Hz}$.

maximise the likelihood function

$L\left(\lambda_{1}, \ldots, \lambda_{n_{\mathrm{f}}}, \beta\right)=\prod_{i=1}^{n_{f}} \prod_{j=1}^{n_{j}} f_{X_{j}}^{\max }\left(x_{i j} \mid x_{\max (i)}, t_{i j}\right)$,

where $x_{i j}=\ln \left[a_{j}\left(f_{i}\right)\right]$ with $a_{j}\left(f_{i}\right)$ the largest acceleration at spectral frequency $f_{i}$ experienced at the site of interest within the time interval $t_{i j}$, and $j=1, \ldots, n_{i}$. The value $n_{i}$ denotes the number of respective time intervals and index $i$ denotes the frequency number. It is not difficult to see that Eq. (8) is a simple extension of the likelihood function from 'one dimension' (PGA only) to $n_{\mathrm{f}}$ dimensions, i.e. the number of analyzed spectral frequencies.

\section{Seismic hazard maps for the Japanese islands}

The procedure as described above was used to estimate the seismic hazard parameters [8] in the vicinity of the 

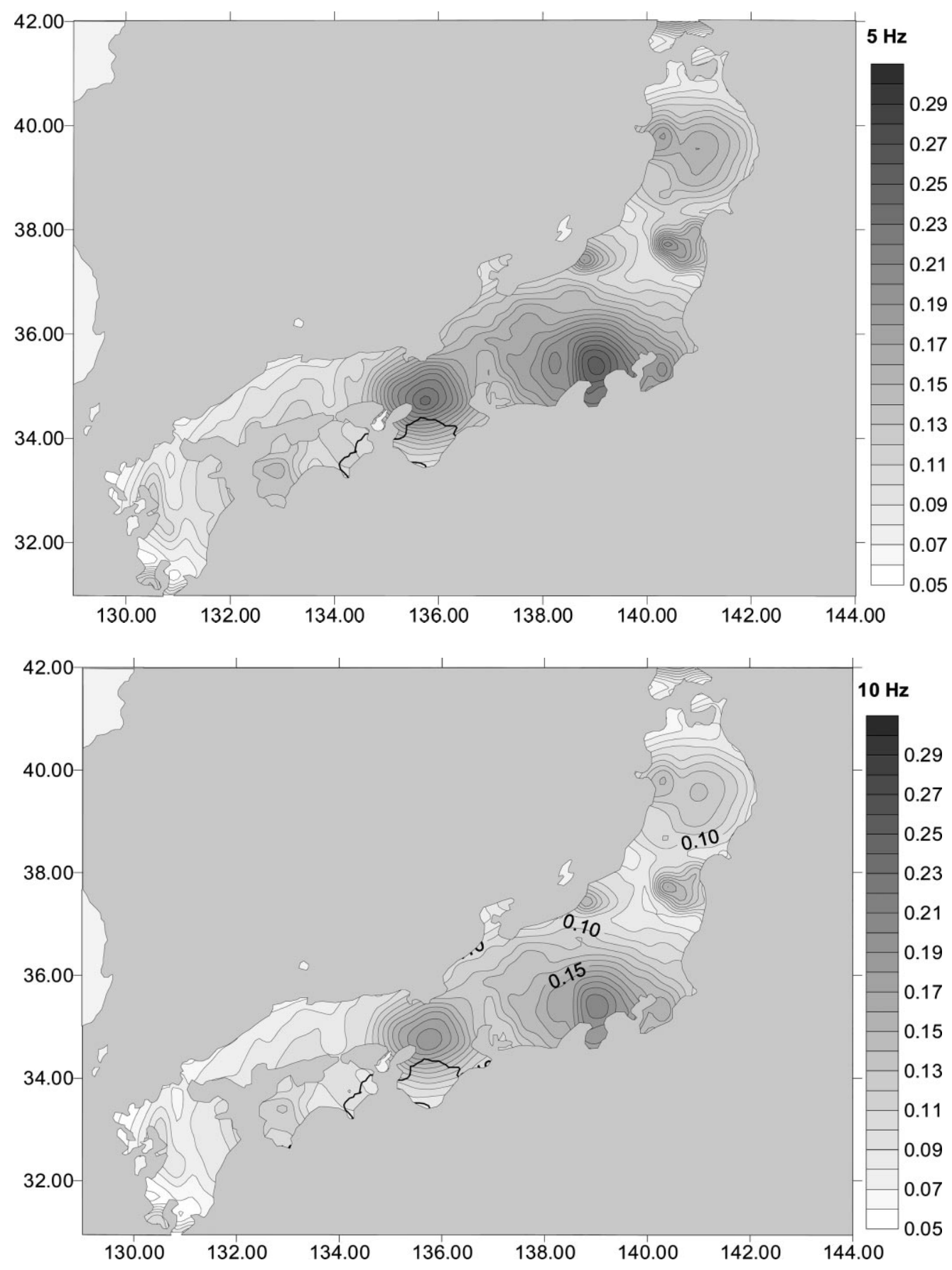

Fig. 3. (continued)

Japanese islands, located between $42^{\circ} \mathrm{N}, 144^{\circ} \mathrm{E}$ and $31^{\circ} \mathrm{N}$, $129^{\circ} \mathrm{E}$ (Fig. 2). The PSHA is based on three seismic databases: the instrumental catalogue compiled from the Japan Meteorological Agency (JMA) data file for the time period between 1926 and 1997; Utsu's [9] catalogue for the time period between 1885 and 1925, and Usami's [10] historical catalogue from 599 to 1884 . Only the main shocks, as selected by the Gardner-Knopoff [11] procedure, were used.

It is assumed that the only reliable information in the assessment of the seismic hazard parameters in the vicinity of the studied region comes from the knowledge of past seismicity. No data were available which could provide a firm basis for identification and grading of seismic source zones. The procedures were applied to seismic data limited to the Japanese islands, where the majority of seismicity appears to be related to the two arc systems called North East and Southwest Japan systems (Fig. 1). The compiled catalogue is incomplete in terms of historic events, but it is natural to expect that information on the largest earthquakes would be more complete and therefore, their distribution 
could be used. Hence, the catalogue is divided into an incomplete part (historic) and three complete parts, for which information from instruments was obtained. The first part of the catalogue contains the largest seismic events (with $M_{\mathrm{S}}$ magnitudes equal to or exceeding $m_{0}=5.2$ ) that occurred during the period 1 January 599-31 December 1884. It was assumed that, for all of these events, the standard deviation of the determination of magnitude was 0.5 . The second part (from 1 January 1885 to 31 December 1925 ) includes the complete catalogue of seismic events, from magnitude level $m_{\min }^{(1)}=5.4$ upwards. The uncertainty of magnitude was assumed to be 0.4 . The third part (from 1 January 1926 to 31 December 1977) includes the complete data, from magnitude level $m_{\min }^{(2)}=4.3$ upwards. The uncertainty of magnitude was assumed to be 0.2 . The fourth part (from 1 January 1978 to 31 December 1997) includes the complete data, from magnitude level $m_{\min }^{(3)}=3.3$ upwards. The uncertainty of magnitude was assumed to be 0.1 . The first part of the catalogue contains 162 of the largest seismic events. The second part contains 73 events, with $M_{\mathrm{S}}$ magnitude 5.4 or larger, and the third contains 1531 events, with $M_{\mathrm{S}}$ magnitude 4.3 or larger. The fourth part contains 2730 events, with $M_{\mathrm{S}}$ magnitude 3.3 or larger.

Application of the $m_{\max }$ estimator [Eq. (3)] for the area surrounding the selected site, gives $\hat{m}_{\max }=8.33 \pm 0.26$, $\hat{\beta}=1.79 \pm 0.01$ (or equivalently $\hat{b}=0.76 \pm 0.01$ ), and $\hat{\lambda}_{A}=199.48 \pm 3.96$. The area-specific mean activity rate $\hat{\lambda}$ was calculated for the $M_{\mathrm{S}}$ magnitude $m_{\min }$ equal to 3.0.

The horizontal component of the PGA at the selected site was calculated using formula (5), where $M$ is the JMA magnitude $\left(M_{\mathrm{S}}=1.27 M_{\mathrm{JMA}}-1.82\right)$. The attenuation curve regression coefficients $c_{1}=0.206, c_{2}=0.477$, $c_{3}=-0.00144, c_{4}=-1.0$ were used after Molas and Yamazaki $[12,13]$. In the original attenuation formula by Molas and Yamazaki, PGA is expressed in terms of $\mathrm{cm} / \mathrm{s}^{2}$.

Fig. 2 depicts the contours of the horizontal component of PGA, determined for the Japanese islands at a grid size of $0.05^{\circ}$, at a $10 \%$ probability in 50 years. Fig. 3 shows the results of the seismic hazard assessment expressed in terms of acceleration response spectra (5\% damped) at the frequencies $1,3,5$ and $10 \mathrm{~Hz}$. The regression coefficients $c_{1 i}, \ldots, c_{4 i}$ of the attenuation Eq. (7) of the response spectra were recalculated from the respective coefficients given by Molas and Yamazaki [13]. These attenuation equations predict the mean values of $\ln (\mathrm{SA})$ (or equivalently the median value of SA), for sites of hard rock.

\section{Discussion and conclusions}

The results in Figs. 2 and 3 show similar spatial patterns of hazard parameters. The spatial changes of the hazard acceleration response parameter are observed to be negatively correlated with frequency. The high values of ground motion parameters are found in central Japan (Honshu) and near to the Fuji Triple Junction, reflecting the high stress due to a bending of the two oceanic plates-the Philippine Plate and the Pacific Plate. Our results are directly consistent with those of Kumomoto [14], who determined the PGA parameter based on trenching data of quaternary faults in Japan.

The technique used above for seismic hazard assessment has been developed specifically for the estimation of seismic hazard at individual sites without the subjective judgment involved in the definition of seismic source zones, in as much as specific active faults have not been identified and mapped and the causes of the seismicity are not well understood.

This technique combines the best features of the 'deductive' [2] and 'historic' [4] procedures. Since the maximum regional magnitude, $m_{\max }$, is of principal importance in any PSHA, the statistical technique to be used for the evaluation of this important parameter is prescribed. This approach is particularly useful in the mapping of seismic hazard in areas where both, historical observations of large events and complete, recent instrumental observations are available. The procedure takes into account the incompleteness of the seismic catalogues.

Our seismic hazard analysis is a preliminary result and only a first step in describing the spatial variability of detailed seismic hazard potential of the Japanese islands. Therefore, the next step would be to investigate for different reginalization based on the changes of seismotectonic characters in the Japanese islands.

\section{Acknowledgements}

We are grateful to Dr Kunhiko Shimazaki, Dr Stefan Wiemer and Dr Xinglin Lei for making the earthquake catalogue available. Also, we thank Dr Kenji Maeda provided trench data and Dr Takashi Shu Kumamoto for sending seismic hazard maps. A.O. Öncel acknowledges the support of AIST fellow during his stay at the Geological Survey of Japan as a visiting scientist.

\section{References}

[1] McGuire RM. Computation of seismic hazard. Ann Di Geofisica 1993;36:181-200.

[2] Cornell CA. Engineering seismic risk analysis. Bull Seism Soc Am 1968;58:1583-606.

[3] Öncel AO. Alptekin. Effect of aftershocks on estimation of earthquake hazard parameters: an example from the north Anatolian fault zone. Natural Hazards 1999;19:1-11.

[4] Veneziano D, Cornell CA, O'Hara T. Historic method for seismic hazard analysis. Elect Power Res Inst, Report, NP-3438, Palo Alto, 1984.

[5] Kijko A, Graham G. 'Parametric-historic' procedure for probabilistic seismic hazard analysis. Part I: Assessment of maximum regional magnitude mmax. Pure and Applied Geophysics 1998;152:413-42.

[6] Kijko A, Graham G. "Parametric-Historic" procedure for probabilistic seismic hazard analysis. Part II: Assessment of seismic hazard at specified site. Pure and Applied Geophysics 1999;154:1-22.

[7] Kijko A, Graham G, Öncel AO. 'Parametric-historic' procedure for seismic hazard assessment and its application to the Istanbul area. 
Abstracts, TMS 2 Hazard Assessment, 21-25 September, Istanbul, 1998.

[8] Kijko A, Sellevoll MA. Estimation of earthquake hazard parameters from incomplete data files. Part II: Incorporation of magnitude heterogeneity. Bull Seism Soc Am 1992;82:120-34.

[9] Utsu T. Catalog of large earthquakes in the region of Japan from 1885 through 1980. Bull Earthq Res Inst, Univ Tokyo 1982;57:401-63.

[10] Usami T. Materials for comprehensive list of destructive earthquakes in Japan. Tokyo: Tokyo Press, 1996.

[11] Gardner JK, Knopoff L. Is the sequence of earthquakes in Southern
California, with aftershocks removed, Poissonian? Bull Seism Soc Am 1974(64):1363-7.

[12] Molas GL, Yamazaki F. Attenuation of earthquake ground motion in Japan including deep focus events. Bull Seism Soc Am 1995;85:11528.

[13] Molas GL, Yamazaki F. Attenuation of response spectra in Japan using new JMA records. Bull ERS 1996;29:115-28.

[14] Kumamoto T. Long-term conditional seismic hazard of quaternary active faults in Japan. J Seismo Soc Japan 1998;50:53-71 (ZISHIN Ser. 2). 